\title{
Patterns of ICU admissions and outcomes in patients with solid malignancies over the revolution of cancer treatment
}

\author{
Clara Vigneron ${ }^{1} \mathbb{0}$, Julien Charpentier ${ }^{1}$, Sandrine Valade², Jérôme Alexandre 3 , Samy Chelabi ${ }^{4}$, \\ Lola-Jade Palmieri ${ }^{5}$, Nathalie Franck ${ }^{6}$, Valérie Laurence ${ }^{7}$, Jean-Paul Mira ${ }^{1,8}$, Matthieu Jamme ${ }^{9,10}$ and \\ Frédéric Pène ${ }^{1,8^{*}}$
}

\begin{abstract}
Background: Major therapeutic advances including immunotherapy and targeted therapies have been changing the face of oncology and resulted in improved prognosis as well as in new toxic complications. The aim of this study is to appraise the trends in intensive care unit (ICU) admissions and outcomes of critically ill patients with solid malignancies. We performed a retrospective single-centre study over a 12-year period (2007-2018) including adult patients with solid malignancies requiring unplanned ICU admission. Admission patterns were classified as: (i) specific if directly related to the underlying cancer; (ii) non-specific; (iii) drug-related or procedural adverse events.

Results: 1525 patients were analysed. Lung and gastro-intestinal tract accounted for the two main tumour sites. The proportion of patients with metastatic diseases increased from 48.6\% in 2007-2008 to 60.2\% in 2017-2018 ( $p=0.004)$. Critical conditions were increasingly related to drug- or procedure-related adverse events, from $8.8 \%$ of ICU admissions in 2007-2008 to 16\% in 2017-2018 ( $p=0.01)$. The crude severity of critical illness at ICU admission did not change over time. The ICU survival rate was $77.4 \%$, without any significant changes over the study period. Among the 1279 patients with complete follow-up, the 1-year survival rate was 33.2\%. Independent determinants of ICU mortality were metastatic disease, cancer in progression under treatment, admission for specific complications and the extent of organ failures (invasive and non-invasive ventilation, inotropes/vasopressors, renal replacement therapy and SOFA score). One-year mortality in ICU-survivors was independently associated with lung cancer, metastatic disease, cancer in progression under treatment, admission for specific complications and decision to forgo life-sustaining therapies.

Conclusion: Advances in the management and the prognosis of solid malignancies substantially modified the ICU admission patterns of cancer patients. Despite underlying advanced and often metastatic malignancies, encouraging short-term and long-term outcomes should help changing the dismal perception of critically ill cancer patients.
\end{abstract}

Keywords: Solid tumour, ICU, Outcome, Drug-related side effects

*Correspondence: frederic.pene@aphp.fr

${ }^{1}$ Service de Médecine Intensive-Réanimation, Hôpital Cochin, Assistance Publique-Hôpitaux de Paris (AP-HP). Centre \& Université de Paris, Paris, France

Full list of author information is available at the end of the article

\begin{abstract}
Background
Oncology represents a striking field of progress in medicine. Continuous improvements in the prognosis of cancer have been ascribed to earlier detection of diseases through extensive population screening, advances in oncologic treatment and improvements in supportive care $[1,2]$. Besides the historical paradigm of tumour elimination by surgery, radiotherapy and cytostatic
\end{abstract}


chemotherapy, a better understanding of oncogenic processes and anti-tumoral immunosurveillance led to new therapeutic approaches targeting actionable oncogenic mutations in tumour cells or their immune microenvironment. Once considered refractory, some advancedstaged and metastatic malignancies have thus become liable to targeted therapies or immunotherapy that may allow a longstanding control of the disease and prolonged survival with acceptable quality of life [3]. However, new treatments mean new toxicity profiles and life-threatening side effects requiring intensive care unit (ICU) admission [4-6].

Cancer is definitely a major risk factor of acute critical illness. Patients with malignancies, mostly with solid tumours, account for about one in seven critically ill patients, with a substantial proportion of metastatic diseases $(40 \%)$ [7]. Hence an international survey reported that $8.8 \%$ and $3.3 \%$ of patients in the ICU had a history of non-metastatic and metastatic solid cancer, respectively [8]. The recent major changes in the landscape of oncology raise the question of the current indications of ICU admission and the related prognosis in critically ill cancer patients. Although recent studies have well addressed these issues in patients with haematological malignancies, data about patients with solid tumours are scarce, with ICU admission policies based on relatively old studies which do not reflect the current prognosis of cancer.

In the light of the growing incidence of cancer and overall improvement in oncologic prognosis, the aim of this study was to appraise the current trends in unplanned ICU admissions and outcomes of critically ill patients with solid malignancies over a 12-year period.

\section{Methods}

\section{Patients and setting}

We performed a retrospective single-centre study in a 24-bed medical ICU located in a tertiary care hospital with comprehensive oncology departments. Patients admitted for surgical reason are usually hospitalized in a separated surgical ICU. From January 2007 to December 2018, adult patients (age $\geq 18$ years) with a diagnosis of solid tumour (known before or during the ICU stay) requiring unplanned ICU admissions were included. Our hospital policy for critically ill oncological patients is based on early ICU admission for the management and the monitoring of organ failures. Non-inclusion criteria were the following: admission to secure a procedure, planned admissions following elective surgery and patients with cancer cured for more than 5 years. For patients with multiple ICU admissions, only the first qualifying ICU stay was considered.

According to French regulations, this study was approved by the ethics committee from the Société de
Réanimation de Langue Française (CE SRLF 17-03) which waived the need for signed consent. Some data on the subgroup of patients with lung cancer were previously reported elsewhere [9].

\section{Data collection}

The following data related to the underlying malignancy were collected: date of diagnosis, primary tumour site, cancer staging (localized, advanced, metastatic), oncologic status according to RECIST (newly diagnosed cancer during ICU stay or within 1 month before ICU admission, partial remission including stable disease, complete remission, progression), oncologic treatment within 3 months before ICU admission (surgery, radiotherapy, cytostatic chemotherapy, targeted therapy [i.e. drug targeting a specific gene mutation or protein], immunotherapy). The severity at ICU admission was assessed by the Sequential Organ Failure Assessment (SOFA) score computed from the first $24 \mathrm{~h}$ [10]. Organ failure supports were collected throughout the ICU stay, including invasive and non-invasive mechanical ventilation, vasopressors/inotropes and renal replacement therapy. Leukopenia was defined as leukocyte count $<1000 /$ $\mathrm{mm}^{3}$. We recorded the decisions to forgo (withholding or withdrawing) life-sustaining therapies (DFLST). The main outcomes were in-ICU survival and one-year survival in ICU survivors with complete follow-up.

\section{Patterns of ICU admissions}

Causes for ICU admissions were classified into three different categories: (i) specific if directly related to the underlying cancer, i.e. metabolic complications (hypercalcemia, tumour lysis syndrome), pulmonary complications (airway obstruction, pleural effusion), urinary tract obstruction or haematologic complications (disseminated intravascular coagulation, hemophagocytic lymphohistiocytosis), pulmonary lymphangitic carcinomatosis, cardiac tamponade, tumoral bleeding and epilepsy related to brain metastasis; (ii) non-specific if the primary cause for ICU admission was a generic complication including infection, venous thrombo-embolism, non-tumoral bleeding, acute ischaemic events (myocardial infarction, stroke, mesenteric ischaemia, and limb ischaemia); (iii) adverse events distributed in drug-related side effects, based on intrinsic and extrinsic imputation, and procedural adverse events.

\section{Statistical analysis}

Continuous variables were expressed as median (interquartile range) and categorical variables as counts (percentages) and were compared over time using Cuzick test and Chi-square test for trend, respectively. 
In order to take into account the competitive risks and the time-dependent bias, the independent predictors of ICU death were addressed in a multivariate Cox causespecific model, by performing a stepwise backward and forward variable selections based on Akaike information criteria $[11,12]$. The model included variables that reached a p value of less than 0.20 in univariate analysis. Owing to the large number of missing data, the performance status was not entered into the model. Proportional hazard assumption was graphically checked and potential interactions were tested in the final model. DFLST was not included for short-term analysis because of self-fulfilling prophecy risk and to avoid immortality time bias. A similar analysis including DFLST was performed in ICU survivors to identify the determinants of one-year vital status.

All tests were two sided, and p values $<0.05$ were considered statistically significant. All analyses were carried out using R 3.5.1 and R Studio (R foundation for Statistical Computing Vienna, Austria).

\section{Results}

\section{Characteristics of ICU admissions}

Between 2007 and 2018, 17,912 patients were admitted to our ICU (Additional file 1: Figure S1), of whom 1525 patients formed the cohort of interest of this study.

Patients' characteristics are described in Tables 1 and 2. Median age was 67 years [59-75] with a majority of men. The two most frequent primary tumour sites were the gastro-intestinal tract (26.0\%) and lung (24.9\%). The number of ICU admissions increased by 76\% between 2007-2008 and 2017-2018, mainly related to patients with lung and gastro-intestinal malignancies, and to a lesser extent with skin cancer. The proportion of patients with metastatic diseases increased from $48.6 \%$ in $2007-2008$ to $60.2 \%$ in $2017-2018$ ( $p=0.004)$. The performance status prior to the acute complication could be accurately collected for 549 patients, and was severely impaired (stage 3 or 4 ) in 137 (25\%) of them. The proportion of patients treated with antineoplastic treatments within the last 3 months increased from $38.5 \%$ in $2007-2008$ to $53.3 \%$ in $2017-2018$ ( $p=0.001$ ) along with a growing use of immunotherapy or targeted therapy (4.4\% in $2007-2008$ to $18.8 \%$ in $2017-2018, p<0.001$ ). Critical conditions were increasingly related to drugor procedure-related adverse events, from $8.8 \%$ of ICU admissions in $2007-2008$ to $16 \%$ in $2017-2018$ ( $p=0.01)$. Among 57 drug-related adverse events, 10 were related to targeted therapies and seven to immune checkpoint inhibitors. The crude severity of critical illness as assessed by the SOFA score at ICU admission did not change over time. Accordingly, further requirements for ventilatory or circulatory supports did not change either, although the use of renal replacement therapy substantially decreased.

\section{Outcomes and prognostic factors}

The ICU survival rate was $77.4 \%$, without any significant changes over the study period. Among the 1279 patients with complete follow-up, the one-year survival rate was $33.2 \%$. Short-term and long-term vital status, according to the type and stage of cancer, are displayed in Fig. 1. Among 137 patients with severely impaired performance status (stage 3 or 4 ), 84 (61\%) and $2(1.4 \%)$ survived the ICU stay and at one year, respectively. DFLST were increasingly taken during the ICU stay and/or at ICU discharge (35.4\% in $2017-2018$ vs. $27.1 \%$ in $2007-$ 2008, $p=0.008)$. In-ICU and one-year mortality rates of patients with DFLST were $62.6 \%$ and $97.2 \%$, respectively.

In multivariate analysis, independent factors associated with ICU mortality were metastatic disease (causespecific hazard (CSH) 1.78 [1.38-2.30], $p<0.001)$, cancer in progression (CSH $1.62[1.28-2.05], p<0.001)$, need for invasive ventilation ( $\mathrm{CSH} 3.73$ [2.45-5.69], $p<0.001$ ), non-invasive ventilation $(\mathrm{CSH} 2.87 \quad$ [1.65-5.01], $p<0.001$ ), inotropes/vasopressors (CSH 1.74 [1.29$2.34], p<0.001$ ) or renal replacement therapy (CSH 1.33 [1.03-1.70], $p=0.03)$, admission for specific complications (CSH 1.33 [1.04-1.72], $p=0.007$ ) and SOFA score (CSH per point $1.02[1.01-1.03], p<0.001)$. Compared to patients with non-lung cancer, those with lung cancer had a worse prognosis in ICU (CSH 1.32 [1.04-1.66], $p=0.02$ ) (Table 3).

We also addressed the determinants of one-year outcome in 934 ICU survivors (Table 4). One-year mortality was independently associated with lung cancer $(\mathrm{CSH}$ 1.44 [1.16-1.78], $p<0.001$ ), metastatic disease (CSH 1.90 [1.53-2.37], $p<0.001$ ), cancer in progression (CSH 1.39 [1.13-1.72], $p=0.002)$, admission for specific complications (CSH 1.75 [1.43-2.15], $p<0.001$ ) and decision to forgo life-sustaining therapies ( $\mathrm{CSH} 3.21$ [2.54-4.06], $p<0.001)$.

\section{Discussion}

The current therapeutic revolution of cancer dramatically challenges the classical prognostic factors of several malignant diseases, but also reveals new patterns of life-threatening side effects, altogether likely to impact on indications for life-sustaining therapies. We report here significant changes in the features of ICU admissions in patients with solid malignancies, owing to the increasing prevalence of patients with advanced stages of diseases and the increased proportion of patients admitted for drug- or procedure-related adverse events. It is noteworthy that both short-term and one-year survival rates were not impaired by the 
Table 1 Changes in patients' underlying characteristics between 2007 and 2018

\begin{tabular}{|c|c|c|c|c|c|c|c|}
\hline Characteristics & $\begin{array}{l}2007-2008 \\
(n=181)\end{array}$ & $\begin{array}{l}2009-2010 \\
(n=230)\end{array}$ & $\begin{array}{l}2011-2012 \\
(n=241)\end{array}$ & $\begin{array}{l}2013-2014 \\
(n=263)\end{array}$ & $\begin{array}{l}2015-2016 \\
(n=291)\end{array}$ & $\begin{array}{l}2017-2018 \\
(n=319)\end{array}$ & $\mathrm{p}$ \\
\hline Proportions of concurrent ICU admissions (\%) & 6.1 & 7.5 & 7.4 & 7.2 & 8.4 & 10.2 & $<0.001$ \\
\hline Age (years) & $70[59-78]$ & $68[59-76]$ & $67[60-75]$ & $66[57-75]$ & $67[59-74]$ & $67[60-74]$ & 0.15 \\
\hline Male gender & $126(69.6)$ & $143(62.2)$ & $152(63.1)$ & $159(60.5)$ & $163(56)$ & $201(63)$ & 0.87 \\
\hline \multicolumn{8}{|l|}{ Non-cancer comorbid conditions } \\
\hline Hypertension & $76(42.0)$ & $97(42.2)$ & $97(40.2)$ & $120(45.6)$ & $131(45.0)$ & $138(43.3)$ & 0.45 \\
\hline Diabetes & $38(21.0)$ & $44(19.1)$ & $31(12.9)$ & $60(22.8)$ & $55(18.9)$ & $61(19.1)$ & 0.88 \\
\hline Cirrhosis & $10(5.5)$ & $18(7.8)$ & $17(7.1)$ & $17(6.5)$ & $19(6.5)$ & $20(6.3)$ & 0.85 \\
\hline Chronic renal failure & $20(11.0)$ & $20(8.7)$ & $18(7.5)$ & $29(11.0)$ & $28(9.6)$ & $22(6.9)$ & 0.34 \\
\hline Chronic heart failure & $8(4.4)$ & $14(6.1)$ & $9(3.7)$ & $23(8.7)$ & $11(3.8)$ & $16(5.0)$ & 0.93 \\
\hline Type of cancer & & & & & & & $<0.001$ \\
\hline Lung & $50(27.6)$ & $55(24.1)$ & $50(20.7)$ & $60(22.8)$ & $69(23.7)$ & $95(29.8)$ & \\
\hline Gastrointestinal & $25(13.8)$ & $44(19.1)$ & $72(29.9)$ & $90(34.2)$ & $86(29.5)$ & $80(25.1)$ & \\
\hline Urologic & $51(28.2)$ & $63(27.4)$ & $48(19.9)$ & $47(17.9)$ & $47(16.2)$ & $58(18.2)$ & \\
\hline Breast & $16(8.8)$ & $29(12.7)$ & $22(9.1)$ & $24(9.1)$ & $34(11.7)$ & $28(8.8)$ & \\
\hline Head and neck & $12(6.6)$ & $11(4.8)$ & $12(5.0)$ & $5(1.9)$ & $7(2.4)$ & $7(2.2)$ & \\
\hline Gynaecologic & $10(5.5)$ & $8(3.5)$ & $13(5.4)$ & $9(3.4)$ & $10(3.4)$ & $13(4.1)$ & \\
\hline Skin & $1(0.6)$ & $3(1.3)$ & $2(0.8)$ & $3(1.1)$ & $10(3.4)$ & $7(2.2)$ & \\
\hline Others & $16(8.9)$ & $17(7.4)$ & $22(9.1)$ & $25(9.5)$ & $28(9.6)$ & $31(9.7)$ & \\
\hline Time from diagnosis to ICU admission (days) & $259[43-722]$ & $237[43-833]$ & 254 [61-967] & 291 [99-1024] & $267[57-914]$ & $264[70-736]$ & 0.23 \\
\hline Stage ${ }^{a}$ & & & & & & & 0.004 \\
\hline Localized & $53(29.3)$ & $66(28.7)$ & $65(27.0)$ & $58(22.1)$ & $70(24.1)$ & $60(18.8)$ & \\
\hline Advanced & $34(18.8)$ & $55(23.9)$ & $50(20.7)$ & $46(17.5)$ & $62(21.3)$ & $67(21.0)$ & \\
\hline Metastatic & $88(48.6)$ & $106(46.1)$ & $125(51.9)$ & $157(59.7)$ & $156(53.6)$ & $192(60.2)$ & \\
\hline Current cancer status & & & & & & & $<0.001$ \\
\hline Newly diagnosed & $62(34.3)$ & $72(31.3)$ & $71(29.5)$ & $58(22.1)$ & $96(33.0)$ & $82(25.7)$ & \\
\hline Partial remission & $19(10.5)$ & $26(11.3)$ & $47(19.5)$ & $70(26.6)$ & $55(18.9)$ & $83(26.0)$ & \\
\hline Complete remission & $26(14.4)$ & $48(20.9)$ & $37(15.4)$ & $37(14.1)$ & $36(12.4)$ & $36(11.3)$ & \\
\hline Progressive & $65(35.9)$ & $80(34.8)$ & $85(35.3)$ & $94(35.7)$ & $102(35.1)$ & $114(35.7)$ & \\
\hline \multicolumn{8}{|l|}{ Recent oncological treatment ( $<3$ months) } \\
\hline Antitumoral drug treatment & $69(38.5)$ & $98(42.8)$ & $115(47.7)$ & $141(53.6)$ & $163(56.0)$ & $170(53.3)$ & 0.001 \\
\hline Immunotherapy and targeted therapy & $8(4.4)$ & $18(7.8)$ & $17(7.1)$ & $35(13.3)$ & $37(12.7)$ & $60(18.8)$ & $<0.001$ \\
\hline First-line treatment & $45(25.0)$ & $58(25.2)$ & $63(26.1)$ & $4(28.1)$ & $91(31.3)$ & $83(26.0)$ & 0.577 \\
\hline Radiotherapy & $13(7.2)$ & $17(7.4)$ & $16(6.7)$ & $22(8.4)$ & $17(5.8)$ & $22(6.9)$ & 0.543 \\
\hline Surgery & $30(16.8)$ & $38(16.5)$ & $35(14.5)$ & $37(14.1)$ & $25(8.6)$ & $47(14.7)$ & 0.184 \\
\hline
\end{tabular}

a Available for 1510 (99\%) patients

Continuous variables are expressed as median (interquartile range) and categorical variables as counts (percentages)

increasing proportions of metastatic diseases over the study period. Moreover, the proportion of organ failure supports did not change over time, meaning that those supports were considered for patients with advanced malignancies. Only renal replacement therapy was less frequent at the end of the study period, likely related to recent changes in practices towards more delayed initiation [13]. Our results suggest that some malignancies formerly viewed as end-stage conditions are actually prone to prolonged survival after recovery from critical illnesses.
The increasing prevalence of patients with metastasis is associated with a growing proportion of patients under immunotherapy or targeted molecules. Patients with advanced diseases no more eligible to cytostatic chemotherapy (e.g. metastatic melanoma) may still remain liable to alternative treatments resulting in prolonged progression-free and overall survival. Considering the therapeutic options and the predicted lifespan of cancer patients is paramount to the decision-making process of life-sustaining therapies. However, one should keep in mind that data supporting our prognostic assessment 
Table 2 Changes in characteristics and outcomes of ICU admissions between 2007 and 2018

\begin{tabular}{|c|c|c|c|c|c|c|c|}
\hline Characteristics & $\begin{array}{l}2007-2008 \\
(n=181)\end{array}$ & $\begin{array}{l}2009-2010 \\
(n=230)\end{array}$ & $\begin{array}{l}2011-2012 \\
(n=241)\end{array}$ & $\begin{array}{l}2013-2014 \\
(n=263)\end{array}$ & $\begin{array}{l}2015-2016 \\
(n=291)\end{array}$ & $\begin{array}{l}2017-2018 \\
(n=319)\end{array}$ & $p$ \\
\hline \multicolumn{8}{|l|}{ Features at ICU admission } \\
\hline $\begin{array}{l}\text { Time between hospital and ICU admis- } \\
\text { sions (days) }\end{array}$ & $0[0-4]$ & $0[0-3]$ & $0[0-3]$ & $0[0-2]$ & $0[0-3]$ & $0[0-4]$ & 0.625 \\
\hline SOFA score (points) & $5[4-9]$ & $5[4-8]$ & $5[4-8]$ & $5[4-8]$ & $5[4-7.50]$ & $5[4-9]$ & 0.11 \\
\hline Leukopenia & $4(2.2)$ & $9(3.9)$ & $15(6.2)$ & $13(4.9)$ & $15(5.2)$ & $17(5.3)$ & 0.05 \\
\hline \multicolumn{8}{|l|}{ Distribution of acute complications } \\
\hline Non-specific & $113(62.4)$ & $147(63.9)$ & $147(61.0)$ & $174(66.2)$ & $179(61.5)$ & $193(60.5)$ & 0.56 \\
\hline Infection & $58(32.0)$ & $66(28.7)$ & $75(31.1)$ & $91(34.6)$ & $104(35.7)$ & $98(30.7)$ & \\
\hline Bleeding & $6(3.3)$ & $10(4.3)$ & $9(3.7)$ & $17(6.5)$ & $18(6.2)$ & $19(6.0)$ & \\
\hline Ischaemic events & $3(1.7)$ & $2(0.9)$ & $2(0.8)$ & $1(0.4)$ & $4(1.4)$ & $3(0.9)$ & \\
\hline Venous thrombo-embolism & $4(2.2)$ & $8(3.5)$ & $7(2.9)$ & $9(3.4)$ & $6(2.1)$ & $11(3.4)$ & \\
\hline Miscellaneous & $40(22.1)$ & $61(26.5)$ & $54(22.4)$ & $56(21.3)$ & $47(16.2)$ & $62(19.4)$ & \\
\hline Specific & $52(28.7)$ & $62(27.0)$ & $71(29.5)$ & $65(24.7)$ & $84(28.9)$ & $75(23.5)$ & 0.26 \\
\hline Metabolic & $3(1.7)$ & $9(3.9)$ & $9(3.7)$ & $7(2.7)$ & $8(2.7)$ & $5(1.6)$ & \\
\hline Respiratory & $20(11.0)$ & $9(3.9)$ & $9(3.7)$ & $15(5.7)$ & $6(2.1)$ & $10(3.1)$ & \\
\hline Urinary tract obstruction & $13(7.2)$ & $8(3.5)$ & $12(5.0)$ & $6(2.3)$ & $12(4.1)$ & $3(0.9)$ & \\
\hline Haematologic & $0(0.0)$ & $2(0.9)$ & $0(0.0)$ & $0(0.0)$ & $2(0.7)$ & $0(0.0)$ & \\
\hline Others & $16(8.8)$ & $34(14.8)$ & $41(17.0)$ & $37(14.1)$ & $58(19.9)$ & $57(17.9)$ & \\
\hline Adverse events & $16(8.8)$ & $21(9.1)$ & $23(9.5)$ & $24(9.1)$ & $28(9.6)$ & $51(16.0)$ & 0.01 \\
\hline Drug-related adverse events & $5(2.8)$ & $7(3.0)$ & $5(2.1)$ & $10(3.8)$ & $16(5.5)$ & $14(4.4)$ & \\
\hline Procedural adverse events & $11(6.1)$ & $14(6.1)$ & $18(7.5)$ & $14(5.3)$ & $12(4.1)$ & $37(11.6)$ & \\
\hline \multicolumn{8}{|l|}{ Management during the ICU stay } \\
\hline \multicolumn{8}{|l|}{ Organ failure supports } \\
\hline Invasive mechanical ventilation & $80(44.2)$ & $100(43.5)$ & $99(41.1)$ & $101(38.4)$ & $113(38.8)$ & $135(42.3)$ & 0.415 \\
\hline Non-invasive mechanical ventilation & $29(16.0)$ & $20(8.7)$ & $21(8.7)$ & $28(10.6)$ & $23(7.9)$ & $42(13.2)$ & 0.72 \\
\hline Vasopressors/inotropes & $74(40.9)$ & $79(34.3)$ & $81(33.6)$ & $75(28.5)$ & $96(33.0)$ & $107(33.5)$ & 0.14 \\
\hline Renal replacement therapy & $48(26.5)$ & $44(19.1)$ & $43(17.8)$ & $39(14.8)$ & $50(17.2)$ & $35(11.0)$ & $<0.001$ \\
\hline Length of stay in the ICU (days) & $3[1-8]$ & $3[1-6]$ & $2[1-6]$ & $2[1-4]$ & $3[1-5]$ & $3[1-6]$ & 0.91 \\
\hline Decision to forgo life-sustaining therapy & $49(27.1)$ & $62(27.0)$ & $51(21.2)$ & 70 (26.6) & $85(29.2)$ & $113(35.4)$ & 0.008 \\
\hline \multicolumn{8}{|l|}{ Outcomes } \\
\hline ICU survival & $126(69.6)$ & $176(76.5)$ & $203(84.2)$ & $203(77.2)$ & $232(79.7)$ & $240(75.2)$ & 0.407 \\
\hline Hospital survival $^{a}$ & $83 / 168(49.4)$ & $112 / 203(55.2)$ & $136 / 224(60.7)$ & $151 / 250(60.4)$ & 176/281 (62.6) & 166/298 (55.7) & 0.75 \\
\hline One-year survival ${ }^{b}$ & $44 / 146(30.1)$ & $60 / 183(32.8)$ & $70 / 202(34.7)$ & $77 / 223(34.5)$ & $88 / 255(34.5)$ & $85 / 270(31.5)$ & 0.68 \\
\hline
\end{tabular}

a In-hospital survival status was available for 1424 patients

b Survival status at one year was available for 1279 patients

Continuous variables are expressed as median (interquartile range) and categorical variables as counts (percentages)

are generally drawn from relatively fit and somewhat selected patients, with the natural bias of retaining the more optimistic results. Whether these findings apply to patients in the aftermath of critical illness is highly questionable, with respect to impaired nutritional, functional and cognitive status and persistent chronic organ dysfunctions. We strongly advocate a decision-making process where admission and limitation policies are based on close interactions between intensivists and oncologists to confront the theoretical prognosis to the clinical situation, to delineate the realistic short-term and long-term objectives of ICU admission and thereby to provide fair and accurate assessments of the expected benefits and harms of ICU admissions.

Our results are consistent with a recent meta-analysis that reported improved short-term prognosis in solid cancer patients admitted to ICU and with the study of Ostermann et al. [14, 15]. This lack of survival improvement in our study could be explained by significant changes in the oncological characteristics of ICU-admitted patients. In the present study, cancer patients sustained encouraging short-term and long-term survival 

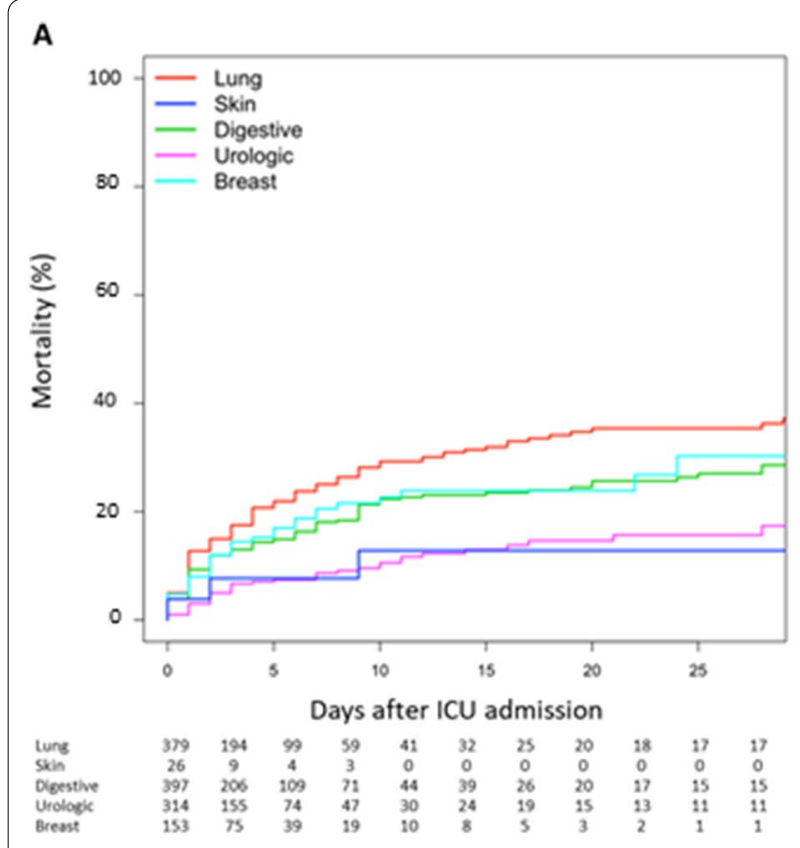

C

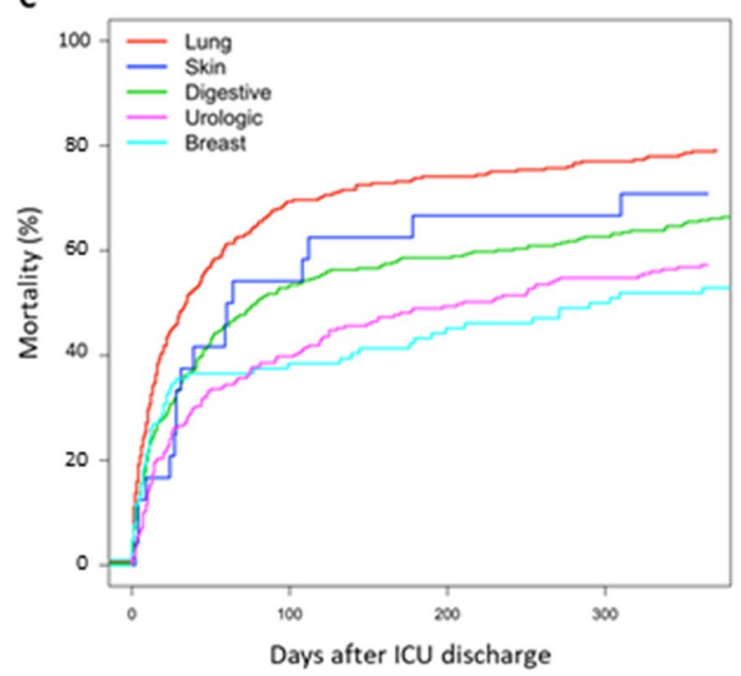

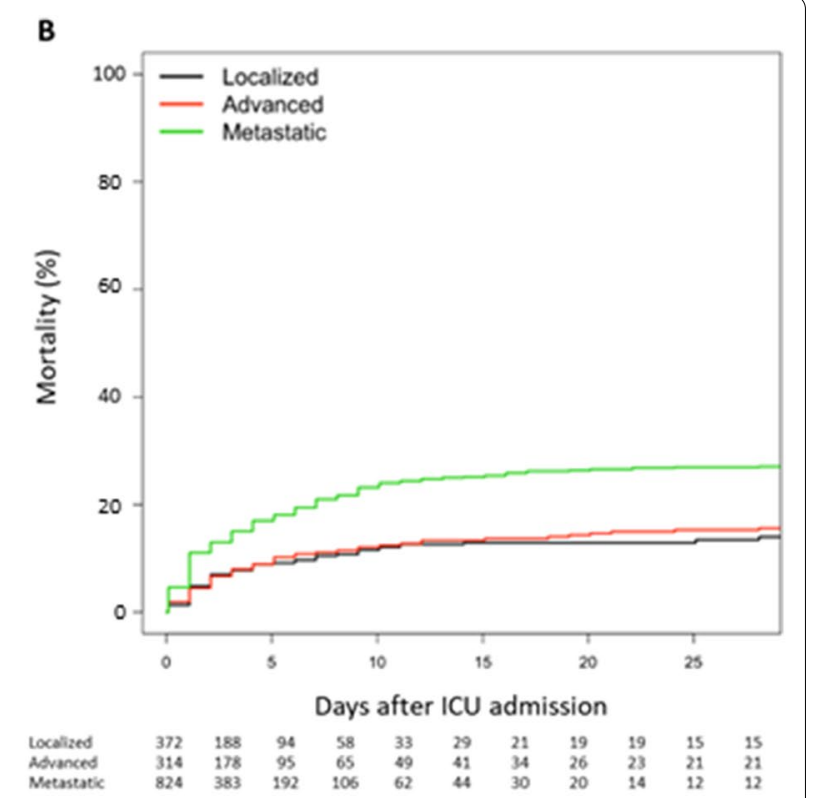

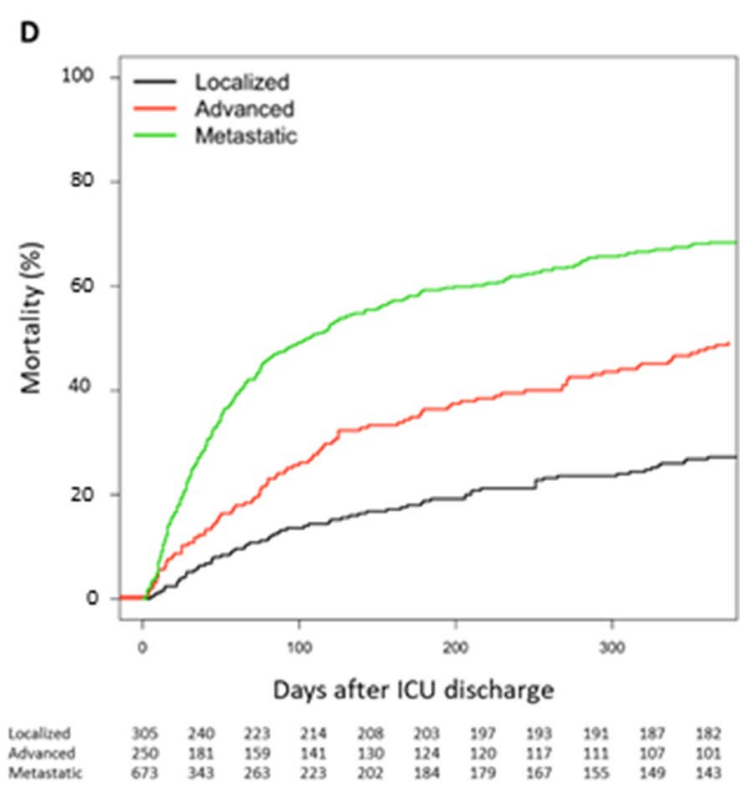

B

$\begin{array}{lccccccccccc}\text { Localized } & 372 & 188 & 90 & 58 & 33 & 29 & 21 & 19 & 19 & 15 & 15 \\ \text { Asvanced } & 314 & 178 & 95 & 65 & 49 & 41 & 34 & 26 & 23 & 21 & 21 \\ \text { Metastatic } & 824 & 383 & 192 & 106 & 62 & 44 & 30 & 20 & 14 & 12 & 12\end{array}$

Fig. 1 Short-term and one-year survival status. 28-day mortality was assessed from ICU admission according to the type (A) and stage (B) of cancer. One-year mortality was assessed in ICU-survivors according to the type (C) and stage (D) of cancer. The landmark is set at the time of ICU discharge. Patients with alternative types of solid tumours are not displayed in this figure

rates, despite the increase in the proportion of patients at metastatic stage over the study period. This suggests that patients with advanced-stage diseases admitted in the ICU were still carefully selected. Determinants of ICU outcome were mostly related to the extent of organ failures, and also to the type and stage of malignancies which account for paramount factors in the decision-making process of therapeutic limitations. Both short-term and long-term prognoses are highly dependent on the casemix of patients, and especially on the proportion of highrisk malignancies [16-18], and we herein pointed the particular burden of lung cancer amongst other malignancies [9]. Determinants of one-year outcome in ICU survivors were related to the stage of malignancies and to the type of complications that warranted ICU admission. Progression in underlying malignancy was linked to 
Table 3 Determinants of ICU mortality

\begin{tabular}{|c|c|c|c|c|c|}
\hline \multirow[t]{2}{*}{ Characteristics } & \multirow{2}{*}{$\begin{array}{l}\text { Survivors } \\
(n=1180)\end{array}$} & \multirow{2}{*}{$\begin{array}{l}\text { Deceased } \\
(n=345)\end{array}$} & \multirow[t]{2}{*}{$p$} & \multicolumn{2}{|l|}{ Multivariate analysis } \\
\hline & & & & $\begin{array}{l}\text { Cause-specific hazard } \\
{[95 \% \mathrm{Cl}]}\end{array}$ & $p$ \\
\hline Age (years) & 67 [59-75] & $68[60-74]$ & 0.7 & & \\
\hline Male gender & $714(60.5)$ & $230(66.7)$ & 0.17 & & \\
\hline \multicolumn{6}{|l|}{ ICU admission } \\
\hline $2007-2012$ & $505(39.1)$ & 147 (43.6) & 0.13 & & \\
\hline 2013-2018 & $675(60.9)$ & $198(57.4)$ & & & \\
\hline Type of cancer & & & $<0.001$ & & \\
\hline Lung & $257(21.8)$ & $122(35.4)$ & & & \\
\hline Gastrointestinal & $298(25.3)$ & $99(28.7)$ & & & \\
\hline Urologic & $275(23.3)$ & $39(11.3)$ & & & \\
\hline Cutaneous & $23(2.0)$ & $3(0.9)$ & & & \\
\hline Breast & $123(10.4)$ & $30(8.7)$ & & & \\
\hline Lung cancer & $257(21.8)$ & $122(35.4)$ & $<0.001$ & $1.32[1.04-1.66]$ & 0.02 \\
\hline Non-lung cancer & $923(78.2)$ & $223(64.6)$ & & - & - \\
\hline Stage & & & $<0.001$ & & \\
\hline Localized & $314(26.6)$ & $58(16.8)$ & & - & - \\
\hline Advanced & $258(21.9)$ & $56(16.2)$ & & - & - \\
\hline Metastatic & $597(50.6)$ & $227(65.8)$ & & $1.78[1.38-2.30]$ & $<0.001$ \\
\hline Status & & & $<0.001$ & & \\
\hline Newly diagnosed & $314(26.6)$ & $105(30.4)$ & & - & - \\
\hline Partial remission & $259(21.9)$ & $41(11.9)$ & & - & - \\
\hline Complete remission & $182(15.4)$ & $38(11.0)$ & & - & - \\
\hline In progression & $384(32.5)$ & $156(45.2)$ & & $1.62[1.28-2.05]$ & $<0.001$ \\
\hline \multicolumn{6}{|l|}{ Treatment in the past 3 months } \\
\hline Chemotherapy & $575(48.7)$ & $181(52.5)$ & 0.215 & - & - \\
\hline Surgery & $183(15.5)$ & $29(8.4)$ & 0.003 & $0.68[0.45-1.01]$ & 0.05 \\
\hline Immunotherapy/targeted therapy & $129(10.9)$ & $46(13.3)$ & 0.256 & - & - \\
\hline SOFA score & $5[4-7]$ & $8[5-12]$ & $<0.001$ & $1.02[1.01-1.03]^{\mathrm{a}}$ & $<0.001$ \\
\hline \multicolumn{6}{|l|}{ Organ failure supports } \\
\hline Invasive mechanical ventilation & $338(28.6)$ & $290(84.1)$ & $<0.001$ & $3.73[2.45-5.69]$ & $<0.001$ \\
\hline Non-invasive mechanical ventilation & $142(12.0)$ & $21(6.1)$ & 0.002 & $2.87[1.65-5.01]$ & $<0.001$ \\
\hline Inotropes/vasopressors & $265(22.5)$ & 247 (71.6) & $<0.001$ & $1.74[1.29-2.34]$ & $<0.001$ \\
\hline Renal replacement therapy & $144(12.2)$ & $115(33.3)$ & $<0.001$ & $1.33[1.03-1.70]$ & 0.03 \\
\hline \multicolumn{6}{|l|}{ Admission patterns } \\
\hline Non-specific complications & $729(61.8)$ & $224(64.9)$ & 0.318 & - & - \\
\hline Specific complications & $313(26.5)$ & $96(27.8)$ & 0.681 & $1.33[1.04-1.72]$ & 0.007 \\
\hline Adverse event complications & $138(11.7)$ & $25(7.2)$ & 0.024 & - & - \\
\hline Drug-related adverse event & $51(4.3)$ & $6(1.7)$ & 0.039 & & \\
\hline Procedural adverse event & $87(7.4)$ & $19(5.5)$ & 0.281 & & \\
\hline
\end{tabular}

${ }^{a}$ The CSH is related to 1-point increase in the SOFA score

worse short- and long-term prognosis. It is well known that specific neoplastic complications, i.e. directly driven by cancer through compression or infiltration of anatomic structures and paraneoplastic syndromes, are associated with a worse prognosis, since poorly reversible despite aggressive chemotherapy [19, 20]. Some malignancies with actionable oncogenic mutations, including a subset of lung adenocarcinoma, are amenable to fast-acting targeted therapies such as tyrosine-kinase inhibitors $[21,22]$. Some clinical conditions with rapidly threatening acute organ failures may even prompt the empirical institution of targeted drugs pending final molecular characterization. In contrast, the slow mechanism of action of immunotherapy, dependent on potent activation of 
Table 4 Determinants of one-year mortality in ICU-survivors

\begin{tabular}{|c|c|c|c|c|c|}
\hline \multirow[t]{2}{*}{ Characteristics } & \multirow{2}{*}{$\begin{array}{l}\text { Alive } \\
(n=424)\end{array}$} & \multirow{2}{*}{$\begin{array}{l}\text { Deceased } \\
(n=510)\end{array}$} & \multirow[t]{2}{*}{$p$} & \multicolumn{2}{|l|}{ Multivariate analysis } \\
\hline & & & & $\begin{array}{l}\text { Cause-specific hazard } \\
{[95 \% \mathrm{Cl}]}\end{array}$ & $p$ \\
\hline Age (years) & $67[59-74]$ & $68[60-76]$ & 0.18 & $1.008[1.007-1.012]$ & 0.03 \\
\hline Male gender & $248(58.5)$ & $297(58.2)$ & 0.4 & & \\
\hline \multicolumn{6}{|l|}{ ICU admission } \\
\hline 2007-2012 & $174(42.1)$ & $210(42.2)$ & 0.54 & & \\
\hline 2013-2018 & $250(58.1)$ & $300(58.8)$ & & & \\
\hline Type of cancer & & & $<0.001$ & & \\
\hline Lung & $65(15.3)$ & $139(27.3)$ & & & \\
\hline Gastrointestinal & $116(27.4)$ & $141(27.7)$ & & & \\
\hline Urologic & $103(24.3)$ & $109(21.4)$ & & & \\
\hline Cutaneous & $7(1.7)$ & $14(2.8)$ & & & \\
\hline Breast & $48(11.3)$ & $26(5.1)$ & & & \\
\hline Lung cancer & $65(15.3)$ & $139(27.3)$ & $<0.001$ & $1.44[1.16-1.78]$ & $<0.001$ \\
\hline Non-lung cancer & $359(84.7)$ & $371(72.7)$ & & - & - \\
\hline Stage & & & $<0.001$ & & \\
\hline Localized & $181(42.7)$ & $73(14.3)$ & & - & - \\
\hline Advanced & $99(23.3)$ & $104(20.4)$ & & - & - \\
\hline Metastatic & $141(33.3)$ & $329(64.5)$ & & $1.90[1.53-2.37]$ & $<0.001$ \\
\hline Status & & & $<0.001$ & & \\
\hline Newly diagnosed & $112(26.4)$ & $164(32.2)$ & & - & - \\
\hline Partial remission & $118(27.8)$ & $80(15.7)$ & & - & - \\
\hline Complete remission & $116(27.4)$ & $34(6.7)$ & & - & - \\
\hline In progression & $78(18.4)$ & $232(45.5)$ & & $1.39[1.13-1.72]$ & 0.002 \\
\hline \multicolumn{6}{|l|}{ Treatment in the past 3 months } \\
\hline Chemotherapy & $172(40.5)$ & $272(53.3)$ & $<0.001$ & - & - \\
\hline Surgery & $93(21.9)$ & $55(10.8)$ & 1.000 & $0.78[0.57-1.05]$ & 0.11 \\
\hline Immunotherapy/targeted therapy & $32(7.5)$ & $72(14.1)$ & 0.002 & - & - \\
\hline SOFA score & $5[3.75-7]$ & $5[4-7]$ & 0.379 & $1.03[0.99-1.06]^{\mathrm{a}}$ & 0.05 \\
\hline \multicolumn{6}{|l|}{ Organ failure supports } \\
\hline Invasive mechanical ventilation & $121(28.5)$ & $140(27.5)$ & 0.519 & - & - \\
\hline Non-invasive mechanical ventilation & $54(12.7)$ & $59(11.6)$ & 0.657 & - & - \\
\hline Inotropes/vasopressors & $91(21.5)$ & $111(21.8)$ & 0.975 & - & - \\
\hline Renal replacement therapy & $49(11.6)$ & $64(12.5)$ & 0.717 & - & - \\
\hline \multicolumn{6}{|l|}{ Admission patterns } \\
\hline Non-specific complications & $292(68.9)$ & $288(56.5)$ & $<0.001$ & - & - \\
\hline Specific complications & $67(15.8)$ & $178(34.9)$ & $<0.001$ & $1.75[1.43-2.15]$ & $<0.001$ \\
\hline Adverse event complications & $65(15.3)$ & $44(8.6)$ & 0.002 & - & - \\
\hline Drug-related adverse event & $17(4.0)$ & $24(4.7)$ & 0.721 & - & - \\
\hline Procedural adverse event & $48(11.3)$ & $20(3.9)$ & $<0.001$ & - & - \\
\hline Decision to forgo life-sustaining therapy & $11(2.6)$ & $120(23.5)$ & $<0.001$ & $3.21[2.54-4.06]$ & $<0.001$ \\
\hline
\end{tabular}

${ }^{\text {a }}$ The CSH is related to 1-point increase in the SOFA score

anti-tumoral immunity, makes it irrelevant to achieve a fast reversal of specific organ failures. An important finding from this report is the increase in admissions related to drug and procedural adverse events, thereby highlighting the hazards related to emerging cancer treatments, most especially immunotherapy [23].
We acknowledge some limitations. This was a singlecentre study, underlined by the development of a comprehensive oncology project in our hospital over the last two decades, and these results may not be fully transposable elsewhere. The collection of data was retrospective, likely reliable for most clinical features, but definitely less 
accurate for more subjective assessments not systematically collected at admission such as functional status, a relevant prognosis factor in this setting $[24,25]$. We were able to collect the one-year vital status for the majority of patients $(79.2 \%)$ discharged from the ICU, but we could not assess how the critical illness altered the optimal continuation of anticancer treatment, nor the quality of life $[16,26]$.

\section{Conclusion}

Intensive care medicine is committed to support and accompany the progress in medicine. Advances in the management and the prognosis of solid malignancies have substantially modified the ICU admission patterns. Despite underlying advanced and often metastatic malignancies, three out of four patients survived the ICU stay and about one-third remained alive at one year. Such encouraging outcomes should help change the dismal perception of critically ill cancer patients. How critical illness may impact on the long-term prognosis of cancer remains to be investigated.

\section{Abbreviations}

SOFA: Sequential Organ Failure Assessment; ICU: Intensive Care Unit; DFLST: Decisions to forgo life-sustaining therapies; CSH: Cause-specific hazard.

\section{Supplementary Information}

The online version contains supplementary material available at https://doi. org/10.1186/s13613-021-00968-5.

Additional file 1: Figure S1. Flowchart.

\section{Authors' contributions}

CV and FP designed the study and drafted the manuscript. CV and JC collected the data. MJ performed the statistical analysis. MJ, SV, JA, SC, LJP, NF, $\mathrm{VL}$, JPM contributed to data interpretation and analysis, and revised the manuscript for important intellectual content. All authors read and approved the final manuscript.

\section{Funding}

This research did not receive any specific grant.

\section{Availability of data and materials}

The datasets used and/or analysed during the current study are available from the corresponding author on reasonable request.

\section{Declarations}

\section{Ethics approval and consent to participate}

According to French regulations, this study was approved by the ethics committee from the Société de Réanimation de Langue Française (CE SRLF 17-03) which waived the need for signed consent.

\section{Consent for publication}

Not applicable.

\section{Competing interests}

FP: Alexion Pharma (institutional grant), Gilead Sciences (consulting and teaching personal fees); JPM: Roche, Am-Pharma, Fresenius (personal fees); MJ: Alexion Pharma (grant).

\section{Author details}

${ }^{1}$ Service de Médecine Intensive-Réanimation, Hôpital Cochin, Assistance Publique-Hôpitaux de Paris (AP-HP). Centre \& Université de Paris, Paris, France. ${ }^{2}$ Service de Médecine Intensive-Réanimation, Hôpital Saint-Louis, AP-HP. Nord \& Université de Paris, Paris, France. ${ }^{3}$ Département d'oncologie Médicale, Hôpital Cochin, AP-HP. Centre \& Université de Paris, Paris, France. ${ }^{4}$ Service de Pneumologie, Hôpital Cochin, AP-HP. Centre \& Université de Paris, Paris, France. ${ }^{5}$ Service de Gastro-Entérologie et Oncologie Digestive, Hôpital Cochin, AP-HP. Centre \& Université de Paris, Paris, France. ${ }^{6}$ Service de Dermatologie-Vénéréologie, Hôpital Cochin, AP-HP. Centre \& Université de Paris, Paris, France. ${ }^{7}$ Service d'oncologie Médicale, Institut Curie, Paris, France. ${ }^{8}$ UMR8104, Institut Cochin, INSERM U1016, CNRS, Université de Paris, Paris, France. ${ }^{9}$ Service de Médecine Intensive-Réanimation, Hôpital Privé de l'ouest Parisien, Ramsay Générale de Santé, Trappes, France. ${ }^{10}$ INSERM U1018, Centre de Recherche en Epidémiologie et Santé des Populations, Team 5 (EpReC, Renal and Cardiovascular Epidemiology), Université Versailles Saint-Quentin, Villejuif, France.

Received: 13 September 2021 Accepted: 10 December 2021

Published online: 24 December 2021

\section{References}

1. Ferlay J, Colombet M, Soerjomataram I, Dyba T, Randi G, Bettio M, et al. Cancer incidence and mortality patterns in Europe: Estimates for 40 countries and 25 major cancers in 2018. Eur J Cancer. 2018;103:356-87.

2. Siegel RL, Miller KD, Jemal A. Cancer statistics, 2019. CA. 2019;69:7-34.

3. Colonna M, Boussari O, Cowppli-Bony A, Delafosse P, Romain G, Grosclaude $\mathrm{P}$, et al. Time trends and short term projections of cancer prevalence in France. Cancer Epidemiol. 2018:56:97-105.

4. Lemiale V, Meert A-P, Vincent F, Darmon M, Bauer PR, et al. Severe toxicity from checkpoint protein inhibitors: What intensive care physicians need to know? Ann Intensive Care. 2019;9:1.

5. Kroschinsky F, Stölzel F, von Bonin S, Beutel G, Kochanek M, Kiehl M, et al. New drugs, new toxicities: severe side effects of modern targeted and immunotherapy of cancer and their management. Crit Care. 2017;21:89.

6. Martins F, Sofiya L, Sykiotis GP, Lamine F, Maillard M, Fraga M, et al. Adverse effects of immune-checkpoint inhibitors: epidemiology, management and surveillance. Nat Rev Clin Oncol. 2019;16:563-80.

7. Taccone F, Artigas AA, Sprung CL, Moreno R, Sakr Y, Vincent J-L. Characteristics and outcomes of cancer patients in European ICUs. Crit Care. 2009;13:R15.

8. Vincent J-L, Marshall JC, Ñamendys-Silva SA, François B, Martin-Loeches I, Lipman J, et al. Assessment of the worldwide burden of critical illness: the Intensive Care Over Nations (ICON) audit. Lancet Respir Med. 2014;2:380-6.

9. Vigneron C, Charpentier J, Wislez M, Mira J-P, Lefebvre A, Fournel L, et al. Short-term and long-term outcomes of patients with lung cancer and life-threatening complications. Chest. 2021;160:1560-4.

10. Vincent JL, Moreno R, Takala J, Willatts S, de Mendonça A, Bruining H, et al. The SOFA (Sepsis-related Organ Failure Assessment) score to describe organ dysfunction/failure. On behalf of the Working Group on SepsisRelated Problems of the European Society of Intensive Care Medicine. Intensive Care Med. 1996;22:707-10.

11. Resche-Rigon M, Azoulay E, Chevret S. Evaluating mortality in intensive care units: contribution of competing risks analyses. Crit Care. 2006;10:R5.

12. Latouche A, Allignol A, Beyersmann J, Labopin M, Fine JP. A competing risks analysis should report results on all cause-specific hazards and cumulative incidence functions. J Clin Epidemiol. 2013;66:648-53.

13. Gaudry S, Hajage D, Schortgen F, Martin-Lefevre L, Pons B, Boulet E, et al. Initiation strategies for renal-replacement therapy in the intensive care unit. N Engl J Med. 2016;375:122-33.

14. Darmon M, Bourmaud A, Georges Q, Soares M, Jeon K, Oeyen S, et al. Changes in critically ill cancer patients' short-term outcome over the last decades: results of systematic review with meta-analysis on individual data. Intensive Care Med. 2019;45:977-87. 
15. Ostermann M, Ferrando-Vivas P, Gore C, Power S, Harrison D. Characteristics and Outcome of Cancer Patients Admitted to the ICU in England, Wales, and Northern Ireland and National Trends Between 1997 and 2013. Crit Care Med. 2017:45:1668-76.

16. Borcoman E, Dupont A, Mariotte E, Doucet L, Joseph A, Chermak A, et al. One-year survival in patients with solid tumours discharged alive from the intensive care unit after unplanned admission: a retrospective study. J Crit Care. 2020;57:36-41.

17. Vincent F, Soares M, Mokart D, Lemiale V, Bruneel F, et al. In-hospital and day-120 survival of critically ill solid cancer patients after discharge of the intensive care units: results of a retrospective multicenter study $-\mathrm{A}$ Groupe de recherche respiratoire en réanimation en Onco-Hématologie (Grrr-OH) study. Ann Intensive Care. 2018;8:1.

18. Toffart A-C, Minet C, Raynard B, Schwebel C, Hamidfar-Roy R, Diab S, et al. Use of intensive care in patients with nonresectable lung cancer. Chest. 2011;139:101-8.

19. Torres VBL, Vassalo J, Silva UVA, Caruso P, Torelly AP, Silva E, et al. Outcomes in critically ill patients with cancer-related complications. PLoS ONE. 2016;11:e0164537.

20. Zerbib Y, Rabbat A, Fartoukh M, Bigé N, Andréjak C, Mayaux J, et al. Urgent chemotherapy for life-threatening complications related to solid neoplasms. Crit Care Med. 2017:45:e640-8.

21. Tandjaoui-Lambiotte Y, Akrour Y, Gibelin A, Gonzalez F, Stoclin A, Moreau $A S$, et al. Tyrosine kinase inhibitors for acute respiratory failure due to non-small-cell lung cancer involvement in intensive care unit. Chest. 2021. https://doi.org/10.1016/j.chest.2021.06.079).

22. Toffart A-C, Dhalluin X, Girard N, Chouaid C, Audigier-Valette C, Duruisseaux $M$, et al. Patients with advanced lung cancer harboring oncogenic mutations should be admitted to intensive care units. Intensive Care Med. 2015;41:164-5.

23. Joseph A, Simonaggio A, Stoclin A, Vieillard-Baron A, Geri G, Oudard S, et al. Immune-related adverse events: a retrospective look into the future of oncology in the intensive care unit. Ann Intensive Care. 2020;10:143.

24. Zampieri FG, Bozza FA, Moralez GM, Mazza DDS, Scotti AV, Santino MS, et al. The effects of performance status one week before hospital admission on the outcomes of critically ill patients. Intensive Care Med. 2017:43:39-47.

25. van der Zee EN, Noordhuis LM, Epker JL, van Leeuwen N, Wijnhoven $\mathrm{BPL}$, Benoit DD, et al. Assessment of mortality and performance status in critically ill cancer patients: a retrospective cohort study. PLoS ONE. 2021;16:e0252771.

26. Normilio-Silva $K$, de Figueiredo AC, Pedroso-de-Lima AC, Tunes-da-Silva G,Nunes da Silva A, Delgado Dias Levites A, et al. Long-term survival, quality of life, and quality-adjusted survival in critically ill patients with cancer. Crit Care Med. 2016;44:1327-37.

\section{Publisher's Note}

Springer Nature remains neutral with regard to jurisdictional claims in published maps and institutional affiliations.

\section{Submit your manuscript to a SpringerOpen ${ }^{\circ}$ journal and benefit from:}

- Convenient online submission

- Rigorous peer review

- Open access: articles freely available online

- High visibility within the field

- Retaining the copyright to your article 